\title{
OPEN Deletion of the clock gene Period2 (Per2) in glial cells alters mood-related behavior in mice
}

\author{
Tomaz Martini ${ }^{1}$, Jürgen A. Ripperger ${ }^{1}$, Jimmy Stalin ${ }^{2}$, Andrej Kores ${ }^{1}$, Michael Stumpe ${ }^{1}$ \& \\ Urs Albrecht ${ }^{1 \bowtie}$
}

The circadian clock regulates many biochemical and physiological pathways, and lack of clock genes, such as Period (Per) 2, affects not only circadian activity rhythms, but can also modulate feeding and mood-related behaviors. However, it is not known how cell-type specific expression of Per2 contributes to these behaviors. In this study, we find that Per 2 in glial cells is important for balancing mood-related behaviors, without affecting circadian activity parameters. Genetic and adenoassociated virus-mediated deletion of Per2 in glial cells of mice leads to reduced despair and anxiety. This is paralleled by an increase of the GABA transporter 2 (Gat2/Slc6a13) and Dopamine receptor D3 (Drd3) mRNA, and a reduction of glutamate levels in the nucleus accumbens (NAc). Interestingly, neuronal Per2 knock-out also reduces despair, but does not influence anxiety. The change in moodrelated behavior is not a result of a defective molecular clock, as glial Bmal1 deletion has no effect on neither despair nor anxiety. Exclusive deletion of Per2 in glia of the NAc reduced despair, but had no influence on anxiety. Our data provide strong evidence for an important role of glial Per2 in regulating mood-related behavior.
Abbreviations
GPer2 Constitutive glial Per2 KOs (Gfap-Cre ${ }^{+}$Per2 $\left.^{f l f l}\right)$
vGPer2 Viral-mediated glial Per2 KO in adult animals (Gfap-iCre into Per $2^{f l / f l}$ mice)
GNAcPer2 Nucleus accumbens glial Per2 KO (Gfap-iCre into Per $2^{f l / f l}$ mice)
vNPer2 Viral-mediated glial Per2 KO in adult animals (Syn1-iCre into Per $2^{f l / f l}$ mice)
vGBmal1 Viral-mediated glial Bmal1 KO in adult animals (Gfap-iCre into Bmalf ${ }^{f l / f l}$ mice

Most organisms from cyanobacteria to humans have time-keeping mechanisms, termed circadian clocks, which allow adaptation to the $24-\mathrm{h}$ day ${ }^{42}$. At the heart of this regulation lies a transcriptional-translational feedback loop, called the molecular clock. This clock is made up of a set of clock genes. In mammals, the positive arm of the clock mechanism is driven by the protein heterodimer of BMAL and CLOCK. This complex activates transcription of negative elements whose proteins Period (PER) and Cryptochrome (CRY) inhibit their own transcription by inactivating the BMAL/CLOCK transcriptional complex, thereby establishing an autoregulatory feedback loop ${ }^{48}$. The individual cellular clocks are orchestrated in an intricate manner to establish coherent systemic rhythms ${ }^{18}$ that are able to provide output signals to regulate various aspects of physiology and behavior. Some of these outputs regulate mood-related behavior through modulation of neurotransmitter synthesis, uptake and degradation ${ }^{15,23,47}$, and regulation of glucocorticoid signaling ${ }^{37}$. Absence or mutation of various clock genes have been associated with mood-related behaviors in mice and humans ${ }^{15,23,34,38,50}$. In particular, a whole-body mutation of the clock gene Per2 revealed a manic phenotype in the Porsolt's forced swim test (FST), which was associated with reduced dopamine degradation, leading to increased dopamine levels in the nucleus accumbens $(\mathrm{NAc})^{23}$. This is consistent with the view that the pharmacological manipulation of the monoaminergic system regulates mood, explaining in part the pathophysiology of depression ${ }^{36}$. However, current treatments for depression are often inefficient and require weeks of medication before the benefits of treatment can be observed ${ }^{16}$. Since major depression is a leading cause of disability in the western world and one of the main causes of death in adolescents, with a total of 800,000 suicides due to depression annually ${ }^{22}$, new approaches of tackling this debilitating condition are needed. An emerging new target for mood interventions are astrocytes ${ }^{52}$, which are in close metabolic and signaling interplay with neurons ${ }^{2,32}$. In order to ensure this interplay, the two cell populations

${ }^{1}$ Department of Biology, Faculty of Science and Medicine, University of Fribourg, 1700 Fribourg, Switzerland. ${ }^{2}$ Department of Oncology, Microbiology and Immunology, Faculty of Science and Medicine, University of Fribourg, 1700 Fribourg, Switzerland. ${ }^{\varpi}$ email: urs.albrecht@unifr.ch 
Figure 1. Genetic deletion of Per2 in glia leads to depression resistant behavior. (A) Immunohistochemistry of sections from suprachiasmatic nuclei (SCN) of control (left panel) and GPer2 mice (middle and right panels) collected at ZT12. Sections were incubated with antibodies against PER2 (green) and glial fibrillary acidic protein (GFAP), a glial marker (red, left and right panels), or NeuN (red, middle panel), a neuronal marker. White arrows in the control panel (left) indicate overlapping PER2 and GFAP signal (yellow) in glial cells, green indicates neuronal PER2 signal and red astrocytic GFAP. In GPer2 sections, PER2 is mostly detected in neurons that are NeuN-positive, giving rise to the yellow color. In contrast, PER2 (green) is not seen in glial cells (red) of GPer 2 mice, indicating a glial specific deletion of PER2. Scale bar: $100 \mu \mathrm{m}$. (B) Immunohistochemistry of sections from the dorsal striatum (DS) of control (left panel) and GPer2 mice (middle and right panels) collected at ZT12. Sections were stained with DAPI (blue) to reveal cell nuclei and were incubated with antibodies against PER2 (green) and GFAP (red, left and right panels) or NeuN (magenta, middle panel). The yellow color indicates overlapping signal of PER2 and GFAP in control animals (left panel) or PER2 and NeuN in neurons (middle panel). No yellow color in GPer2 sections stained for PER2 and GFAP is observed. Scale bar: $10 \mu \mathrm{m}$. (C) Immobility time in the forced swim test (FST) of GPer2 (GCre+ Per2 $2^{f / f l}$, red) and control (GCre$P e r 2^{f l f l}$, blue) animals are shown $\left(\mathrm{n}=13\right.$ or 11 , respectively, two-tailed t-test, $\left.{ }^{* \star} P<0.01\right)$. (D) FST of the Credriver line $\left(G C r e^{+} P e r 2 W T\right)$ and the Per 2 control line (GCre Per2 WT) are shown (n=6, two-tailed t-test, no significant difference is observed). (E) FST of Per 2 mutant (Per2 $2^{\text {Brdm } 1}$, purple) mice and their littermate controls (WT, green) show a significant difference as previously observed ${ }^{23}(\mathrm{n}=13$ and 8 , respectively, two-tailed t-test, $\left.{ }_{* * *} P<0.001\right)$. (F) Representative swimograms of GPer2 $\left(G C r e^{+} P e r 2^{f l f l}\right.$, red $)$ and control (GCre $e^{-}$Per2 ${ }^{f l f l}$, blue) animals. Note the longer stretches of immobility in the control animals (right panel, blue). (G) Representative swimograms of Per 2 mutant (Per2 ${ }^{\text {Brdm1 } 1}$, purple) mice and their littermate controls (WT, green). Note the longer stretches of immobility in the control animals (right panel, green).

need to be precisely synchronized to each other. Systemic and cellular synchronization is one of the main tasks of the circadian clock $^{18}$ and therefore it is not surprising that astrocytes regulate rhythmic behaviors involving clock genes ${ }^{6,8,26,49}$. However, whether astrocytes that lack clock genes affect mood-related behaviors is not known. Since whole-body Per 2 mutant mice display changes in the reward system ${ }^{1,47}$ and despair perception ${ }^{23}$, we tested mice lacking the Per 2 gene in glial cells, including astrocytes.

To this end, we generated mice lacking Per2 in glial fibrillary acidic protein (GFAP)-positive cells by crossbreeding Per2 floxed mice with a Cre mouse line. In a second approach, we deleted Per2 in GFAP positive cells of adult animals by delivering Cre with an adeno-associated virus to exclude developmental contributions to our experiments. Both models lacking Per2 in Gfap-expressing cells (termed GPer2 and vGPer2) were assessed for despair- and anxiety-related behavior using the FST and O-maze test, respectively. We found that mice from both models display a manic-like phenotype and are less anxious compared to control animals. In contrast to the whole-body Per 2 mutation, which displays a manic phenotype with reduced monoamine oxidase A (Maoa) and elevated dopamine levels ${ }^{23}$, Maoa was normal in the GPer 2 knock-out animals. However, we observed changes in the glutamatergic and GABAergic systems, as well as upregulation of the dopamine receptor D3.

\section{Results}

Deletion of Per2 in glial cells by cross-breeding of mice. In order to study the importance of the Per2 gene in glial cells, we crossed our floxed Per2 $\left(P e r 2^{f / f l}\right)$ animals ${ }^{13}$ with mice expressing the Cre-recombinase under transcriptional control of the human Gfap promoter $(G C r e)^{53}$. In families where the Cre was inherited from the maternal side, $58 \%$ of Cre-negative offspring (out of 80 progeny) showed germline recombination leading to total body Per 2 heterozygous deletion. In comparison, $0 \%$ of paternally inherited Cre (out of 260 progeny; Supplemental table 1) showed any germline recombination, as previously described ${ }^{30}$. Therefore, we used only male Cre-positive mice for matings with female Per $2^{f l f l}$ animals in order to obtain glial-specific deletion of Per2 $\left(\mathrm{GCre}^{+} \mathrm{Per}^{\mathrm{fl} / \mathrm{fl}}\right.$ termed GPer2).

Next, we verified glial deletion of PER2 in brain tissue collected at zeitgeber time (ZT) 12 (where ZT0 is lights on and ZT12 is lights off) of GPer2 mice using immunohistochemistry. Sections of the dorsal part of the suprachiasmatic nuclei (SCN) containing mainly arginine-vasopressin (AVP) neurons displayed immunoreactivity with a PER2 antibody (green) (Fig. 1A). In control animals co-staining with a GFAP antibody (red) showed partially overlapping signal with PER2 leading to yellow and orange staining, indicating PER2 expression in glial cells (white arrows, Fig. 1A, left panel). In GPer 2 mice, the red signal obtained with antibodies against the neuronal marker NeuN almost entirely overlapped with the green PER2 signal, resulting in the yellow/orange color (Fig. 1A, middle panel). This indicated that in the GPer2 animals PER2 is still present in neurons. The PER2 (green) and GFAP (red) signal did not overlap in the GPer2 animals and no yellow color was observed (Fig. 1A, right panel), strongly suggesting that PER2 was absent in glial cells. Hence, the green signal is due to neuronal PER2 expression, suggesting specificity of our approach.

To corroborate these observations, we performed immunohistochemistry in the striatum at ZT12 (Fig. 1B). The signals for PER2 (green) and GFAP (red) overlapped in the cytoplasm (yellow) and not in the nucleus (blue) of control animals (Fig. 1B, left panel). In GPer2 animals, NeuN signal (magenta) overlapped with the PER2 signal (green) resulting in the yellow color (Fig. 1B, middle). Note that there was no green signal observed that could stem from glial PER2 expression. In the GPer2 mice the PER2 signal (green) did not overlap with the GFAP signal (red) and PER2 appeared to be mainly present around the nuclei (blue) of neurons. Overall, our immunohistochemistry data indicate that PER2 was absent from glial cells of GPer2 mice. 


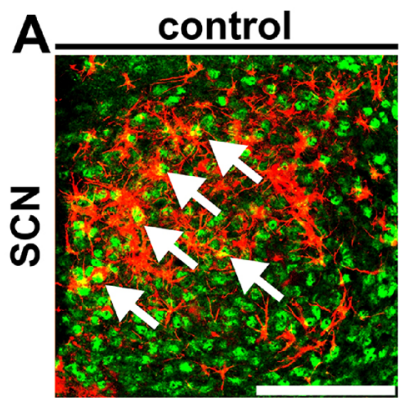

PER2 GFAP

B

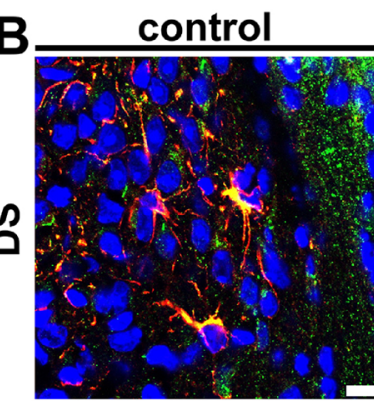

PER2 GFAP DAPI

C

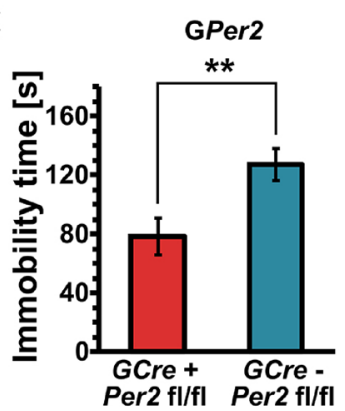

D

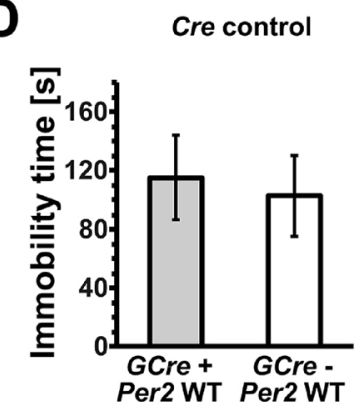

GPer2

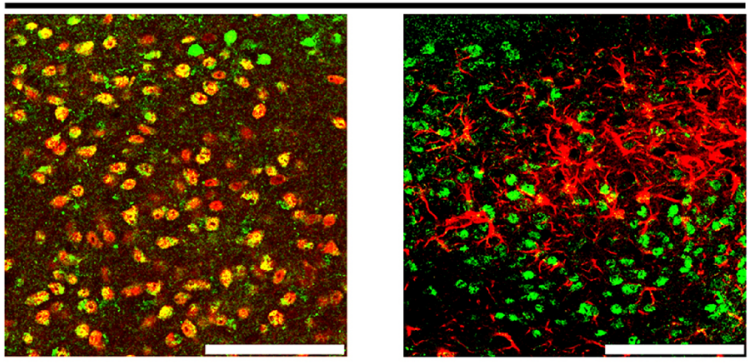

PER2 GFAP

GPer2

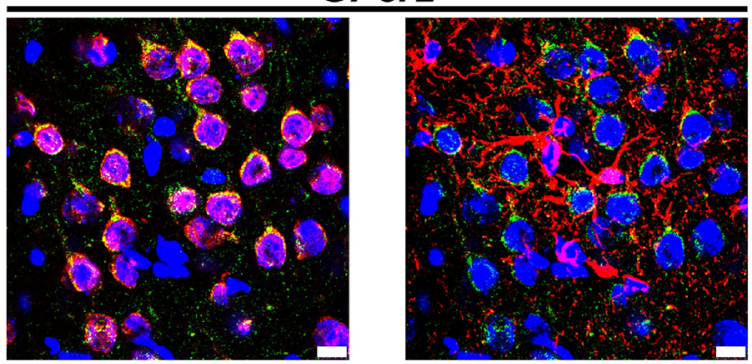

PER2 GFAP DAPI

E total body mutant

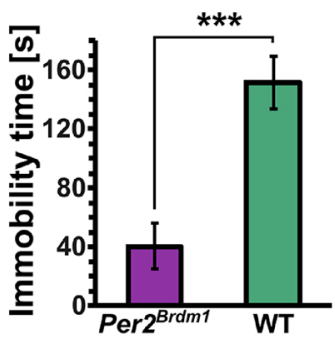

F
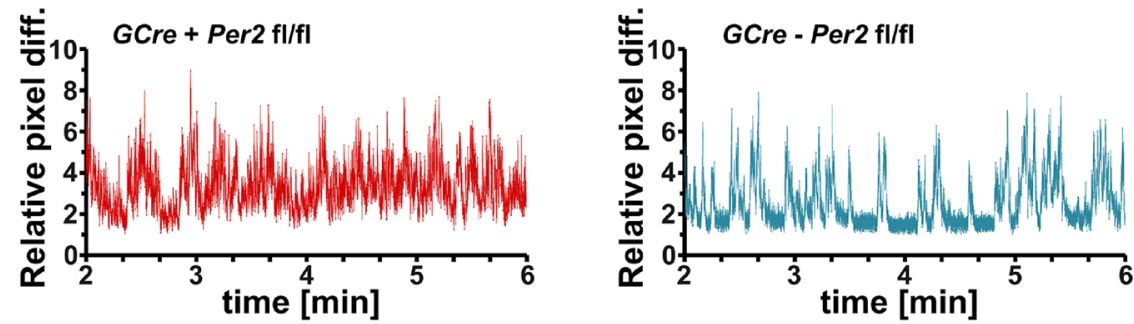

G
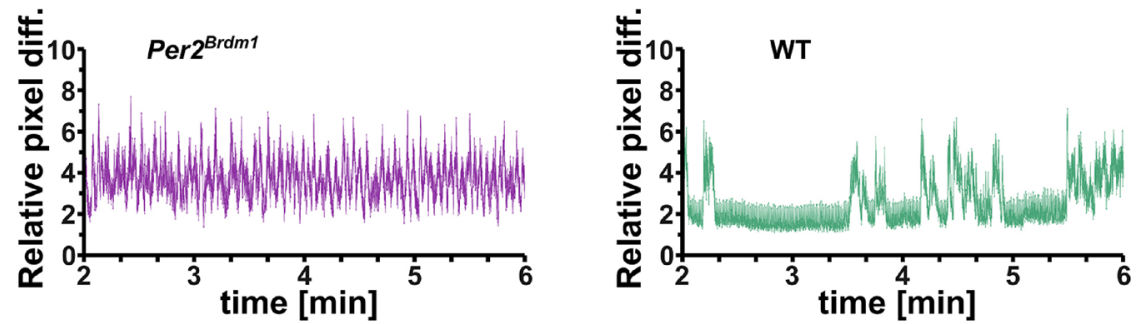
Figure 2. Adeno-associated virus (AAV)-mediated deletion of Per2 in glial cells of adult mice leads to a depression resistant behavior. (A) Fluorescent imaging of whole brains 3 weeks after no injection (left), intravenous (i.v.) injection of the engineered AAV-PHP.eB, which can pass the blood-brain barrier (BBB), containing the general $C A G$ driver (second from left) or the glial Gfap driver (middle). The second to last brain is from an animal with i.v. injected AAV9, which does not pass the BBB, containing the general CAG driver. The last brain (right) is from an animal injected intraperitoneally (i.p.) with the AAV-PHP.eB Gfap-driven construct. Note that only the brains of animals that received the AAV-PHP.eB i.v. display significant fluorescent signal after 3 weeks (orange and yellow color). (B) Fluorescent imaging of whole brains 2 months after injection of the AAVPHP.eB. Note that the fluorescence is still maintained after 2 months post injection and that even the i.p. injected AAV-PHP.eB Gfap is showing signal in the brain now. (C) Sorting of neurons and astrocytes by flow cytometry from brain tissue including the nucleus accumbens (NAc). The left panel shows the removal of debris from a single cell suspension, showing the distribution of debris in the forward as well as in the side scatter (FSC and SSC, respectively). The middle panel shows the removal of $\mathrm{CD}_{1} 1 \mathrm{~b}^{+}$cells (microglia) from the cell suspension. The CD11 $b^{-}$cells (bottom half from middle panel) were then sorted into two distinct cell populations corresponding to astrocytes $\left(\mathrm{GLT1} 1^{+} / \mathrm{CD} 90.2^{-}\right)$and neurons $\left(\mathrm{CD} 90.2^{+} / \mathrm{GLT1} 1^{-}\right.$) (right panel). (D) PCR analysis of astrocytes and neurons from the cell sorting. Microglia $\left(\mathrm{CD} 11 \mathrm{~b}^{+}\right)$as well as astrocytes $\left(\mathrm{GLT} 1^{+} / \mathrm{CD} 90.2^{-}\right)$, but not neurons (CD90.2 $\left.2^{+} \mathrm{GLT}^{-}\right)$from PHP.eB Gfap-iCre infected animals express $i C r e$, indicating that only glia and not neurons could express $i$ Cre in order to delete Per 2 in the Per $2^{f / f l}$ mice. All probes and markers were loaded on the same gel and the resulting photograph was not separated and recomposed. (E) Immunohistochemistry of vGPer2 brain tissue from nucleus accumbens (NAc) isolated at ZT6. The signal for PER2 (green) mainly overlaps with neuronal NeuN signal (red) giving rise to the yellow color. Scale bar: $100 \mu \mathrm{m}$. (F) Immobility time in the forced swim test (FST) of vGPer2 (PHP.eB Gfap-iCre, green) and control (PHP.eB control, blue) animals are shown $\left(\mathrm{n}=7\right.$, two-tailed t-test, $\left.{ }^{* *} P<0.01\right)$. (G) Representative swimograms of vGPer2 (AAV-PHP.eB Gfap$i C r e$, green) and control (AAV-PHP.eB control, blue) animals. Note the longer stretches of immobility in the control animals (right panel, blue).

GPer2 mice display reduced despair. The glial Per2 knock-out animals (GPer2) were tested for despairbased behavior, which is one of the important manifestations of depression. To this end, we used the forced swim test (FST) to assess time of immobility at ZT ${ }^{23}$. We observed that GPer2 mice $\left(G C r e^{+}\right.$Per $\left.2^{f l / f l}\right)$ were significantly less immobile compared to control animals $\left(G C r e^{-} P e r 2^{f l f l}\right)$ (Fig. 1C). The Cre-driver animals used for crossing

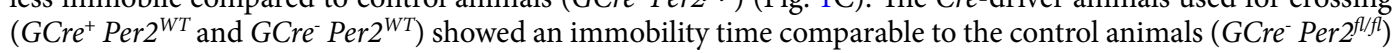
(Fig. 1D). This illustrated that the lower immobility observed in the GPer2 mice was specific to the lack of Per2 in glial cells. As a positive control, we reproduced the lower immobility phenotype of Per $2^{\text {Brdm } 1}$ mutant mice (Fig. 1E) as described previously in male mice ${ }^{23}$, but this time using female animals to demonstrate that the phenotype was not gender specific. Figure $1 \mathrm{~F}$ shows examples of primary data depicted as mobility over time (swimograms) with the corresponding color-coding. The data were obtained using a self-developed movement analysis software to determine mobility in an automated unbiased manner (see methods). It was evident that the control animals displayed long stretches of continuous baseline signal, which corresponded to long time stretches of immobility (Fig. 1F,G, right panels). These long stretches of immobility were very short or absent in both the GPer2 (GCre ${ }^{+}$Per $\left.2^{f l f l}\right)$ and Per $2^{\text {Brdm1 }}$ mutant mice (Fig. 1F,G, left panels). Taken together, our data show that lack of Per2 in glial cells was sufficient to reproduce the manic-like phenotype observed previously in wholebody Per 2 mutant mice ${ }^{23}$. This suggests that Per2 in glial cells may play an important role in the development of despair-based behavior contributing to depression.

Deletion of Per2 in glial cells using adeno-associated virus (AAV)-delivered Cre recombinase. The Gfap-Cre driver mouse line used above has been reported to potentially express Cre in some neuronal progenitor cells $s^{24,40}$. Furthermore, the Cre recombinase is most often inserted randomly into the genome and its correct position and number of copies are unknown. Additionally, Cre leakage was observed in some cases leading to unwanted recombination events ${ }^{29,46}$. Due to these potential problems and to exclude developmental effects in our deletion approach above, we delivered into adult mice a construct expressing the codonimproved Cre-recombinase (iCre) using engineered adeno-associated viruses (AAVs). An engineered AAV for efficient non-invasive gene delivery that can cross the blood-brain barrier (BBB) was used ${ }^{11}$.

This AAV-PHP.eB contained a vector expressing iCre under control of the human Gfap promoter with an enhanced green fluorescent protein $(e G f p)$ as reporter. After intravenous (i.v.) injection via the lateral tail vein, fluorescence in the brain was detectable after 3 weeks in both the synthetic CAG-driven positive control ${ }^{35}$, as well as in the Gfap-driven constructs (Fig. 2A, red and yellow signals). No fluorescence and hence no BBB permeability was detected when using the natural variant AAV9 to deliver the CAG-driven control. Similarly, the non-injected control brain showed only baseline signal (Fig. 2A, brown). Intraperitoneal (i.p.) injection of the AAV-PHP.eB Gfap-driven Cre-construct also showed only baseline signal after 3 weeks. After 2 months, however, even i.p. delivery of the AAV-PHP.eB Gfap-driven construct resulted in detectable fluorescence, comparable to that of the i.v. injection (Fig. 2B, right), which is quite remarkable and is shown here to work for the first time. We termed the AAV mediated deletion of Per 2 in glial cells vGPer2.

Next, we wanted to check if the approach was specific for glial cells (astrocytes and microglia). We used flow cytometry and fluorescence-activated cell sorting to separate microglia $\left(\mathrm{CD} 11 \mathrm{~b}^{+}\right)$, astrocytes $\left(\mathrm{GLT} 1^{+}\right)$and neurons $\left(\mathrm{CD} 90.2^{+}\right)$. In a first step, the cells were depleted from debris (Fig. $2 \mathrm{C}$, left panel), then the CD $11 \mathrm{~b}^{+}$microglia were removed (Fig. 2C, middle panel) and subsequently the GLT1 $1^{+}$astrocytes were separated from the CD90.2 neurons (Fig. 2C, right panel). The presence or absence of $i$ Cre was then determined by PCR on the different 


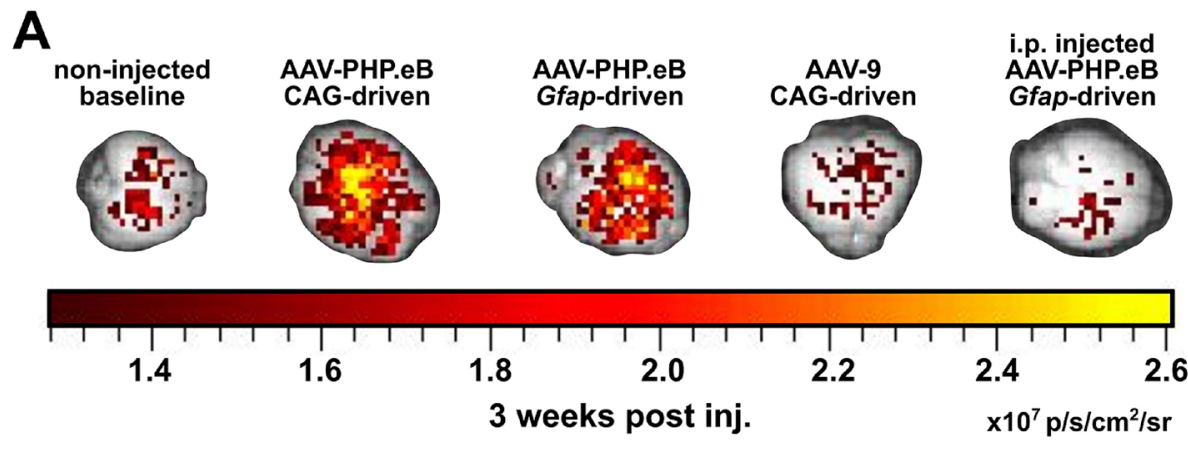

B
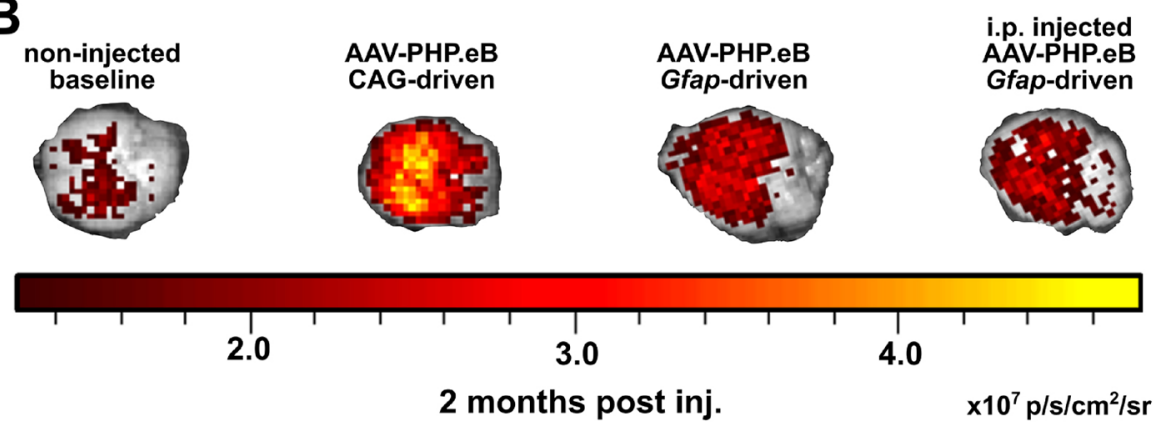

C
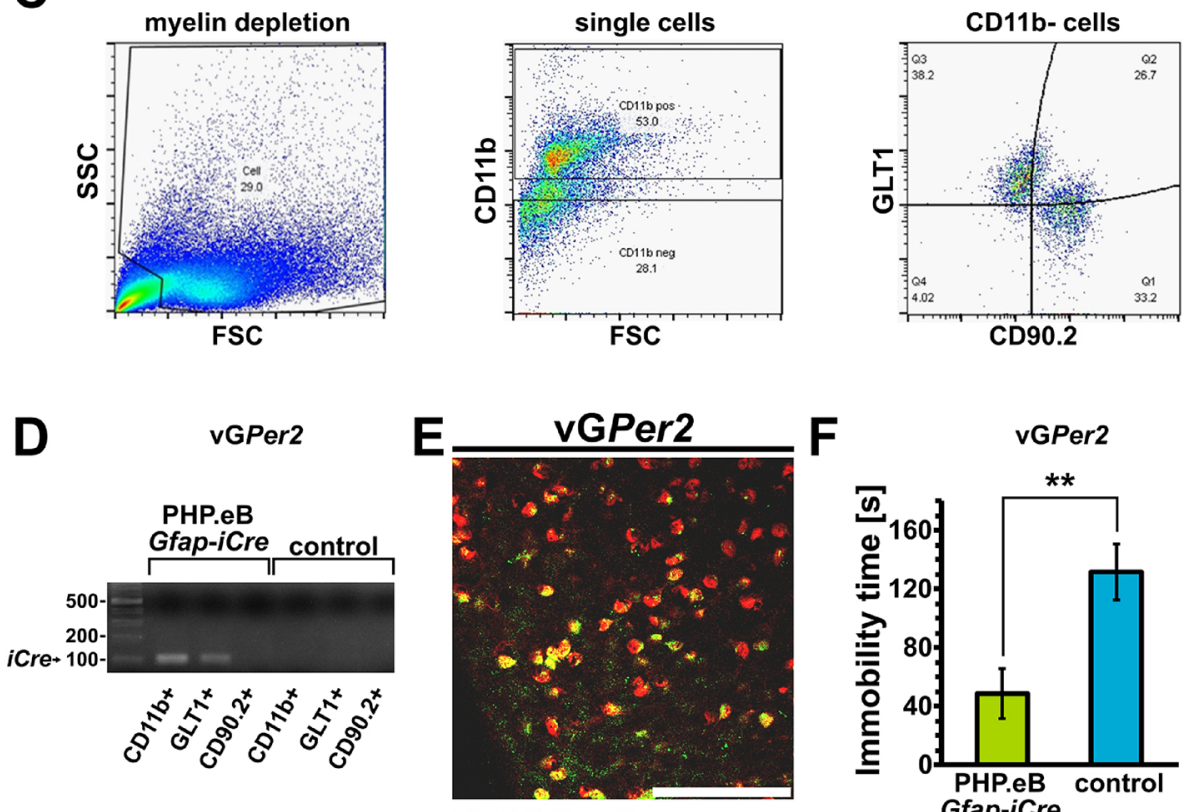

PER2 NeuN
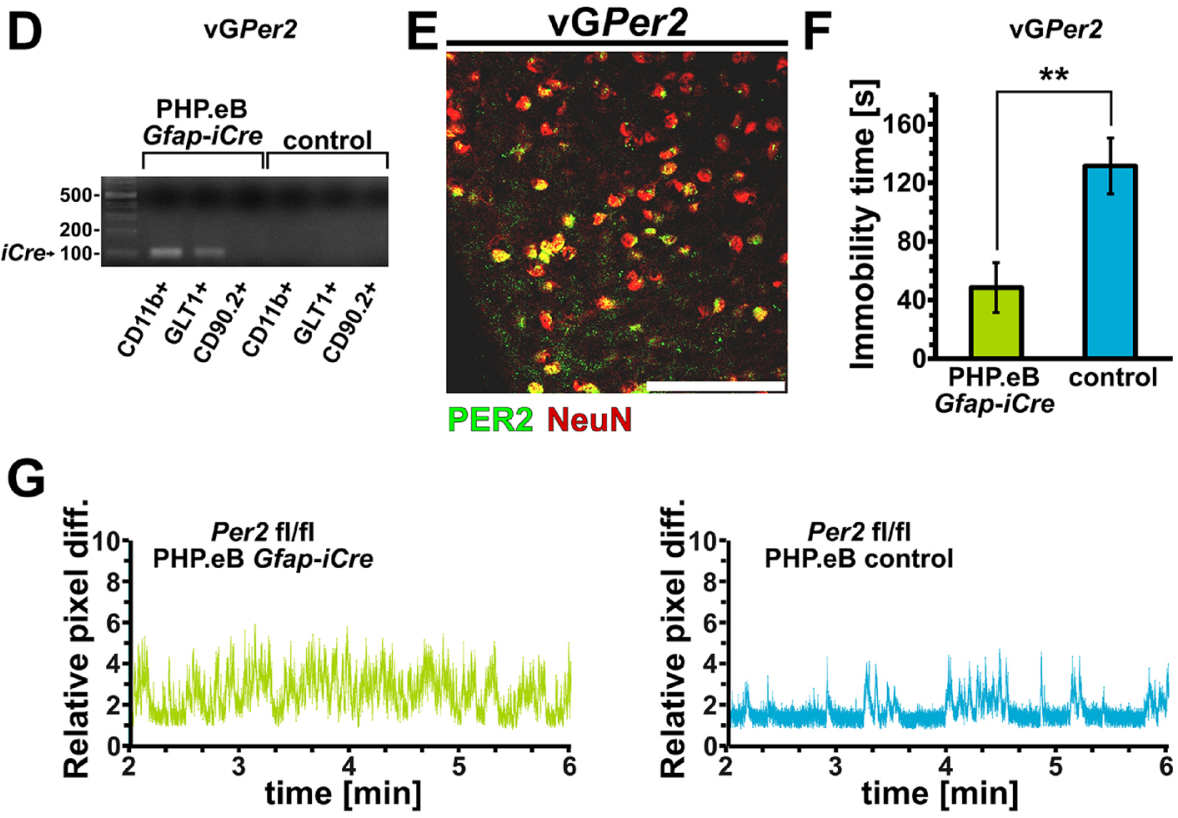
cell populations. We observed that the PHP.eB Gfap-iCre infected microglia $\left(\mathrm{CD} 11 \mathrm{~b}^{+}\right)$and astrocytes $\left(\mathrm{GLT} 1^{+}\right)$ expressed $i$ Cre. In contrast, neurons (CD90.2 $)$ and non-infected controls did not express iCre (Fig. 2D, full size gel in Suppl. Figure 1A). Unfortunately, the sensitivity was not sufficient to reliably detect Per2 expression in WT non-infected controls using this approach. However, immunohistochemistry on nucleus accumbens tissue isolated at ZT6 confirmed that PER2 protein could still be detected in neurons, while outside of neurons PER2 was barely or not detected (Fig. 2E).

vGPer2 mice display reduced despair. The animals with intravenously (i.v.) applied AAV-PHP.eB, which mediated the deletion of Per2 in glial cells (vGPer2), were subjected to the FST in order to assess their immobility in this despair-based behavioral test. We observed that vGPer2 (PHP.eB Gfap-iCre) mice showed significantly lower immobility times compared to control animals, which were injected with a comparable virus, but lacking iCre (PHP.eB control) (Fig. 2F). Examples of swimograms illustrating swimming behavior between minute 2 to 6 are shown (Fig. 2G, Suppl. Figure 2). Longer resting bouts were only detected in control animals (Fig. 2G, right panel, Suppl. Figure 2). This was consistent with the result we obtained by cross-breeding Per2 floxed with Gfap-Cre mice (Fig. 1C, F, G), indicating that the phenotype can be reproduced by deleting Per2 in glial cells of the adult animal. Hence, developmental processes are very unlikely to be responsible for this reduced despair phenotype.

GPer2 and vGPer2 mice show reduced anxiety-like behavior in the O-maze. Depression is a complex state and in addition to despair-related aspects also involves features of anxiety. Therefore, we tested the GPer 2 as well as the vGPer2 animals in the elevated O-maze and measured how much time they spent in the open area of the maze and how many times they entered the open part. We observed that both the GPer2 as well as the vGPer2 animals spent more time in the open section of the O-maze compared to control animals (Fig. 3A, B). Interestingly, both GPer2, as well as vGPer2 mice, displayed a tendency to explore the open sections more frequently (Fig. 3C, D). These observations indicate a reduced anxiety level in mice that lack Per2 in glial cells.

GPer2 mice display normal circadian parameters. Since GPer2 mice display less immobility in the FST and swim more, we tested these animals for the circadian parameters of total activity, circadian period and body temperature fluctuation. We measured running-wheel revolutions in the cages of GPer2 and control animals and observed identical activity profiles with low activity during the $12 \mathrm{~h}$ light phase and high activity during the $12 \mathrm{~h}$ dark phase (Fig. 4A). General activity was measured via an intraperitoneally implanted transmitter that was traced by a detector plate under the floor of the cage. Comparable to the wheel-running activity (Fig. 4A), no difference between the genotypes was observed (Fig. 4B). However, the activity in the second half of the dark phase (ZT16-22) was for both genotypes higher in the wheel-running assessment compared to the general activity pattern. These results show that the reduced immobility time of GPer 2 animals in the FST was not due to a higher activity level compared to controls and, hence, the higher activity in the FST is related to despair rather than to general activity.

Using the wheel-running activity data, we also determined the circadian period $(\tau)$ of the GPer2 and total Per2 knock-out (KO) animals under constant darkness conditions. The circadian period of GPer 2 mice was normal and not significantly different from control animals (Fig. 4C, D, E), indicating that loss of Per2 in glial cells does not affect circadian period. In contrast, whole-body Per $2 \mathrm{KO}$ mice displayed a period shorter than $23 \mathrm{~h}$ (Fig. 4F), consistent with previous findings ${ }^{13,51}$.

Next, we assessed body temperature over $24 \mathrm{~h}$ under a $12 \mathrm{~h}$ light : $12 \mathrm{~h}$ dark cycle (LD 12:12). No significant differences in the body temperature profiles were observed between GPer2 and control animals, in both males (Fig. 4G) as well as females (Fig. $4 \mathrm{H}$ ). This is consistent with the phenotype in Per $2^{\mathrm{Brdm} 1}$ whole body mutant mice at $21{ }^{\circ} \mathrm{C}^{12}$ and liver, neuronal and total body Per 2 knock-out animals ${ }^{13}$.

Taken together, our data illustrate that loss of Per 2 in glial cells does not phenocopy all the characteristics of Per 2 whole-body KO animals, such as a shortening of the free-running period. This highlights a specific role of glial Per2 in mood-related behaviors.

Molecular changes in GPer2 mice. Mood-related behaviors including depression are regulated by a number of different brain nuclei and regions $s^{4,5,7,19,20,54}$. We investigated three of those brain regions, the nucleus accumbens (NAc), the medial prefrontal cortex (mPFC) and the amygdala (AMY), as well as the hypothalamus (HYP) as a control brain region. The aforementioned brain regions also showed altered activity in response to swim stress ${ }^{19}$. We focused our attention on expression of genes involved in the synthesis, reuptake and degradation of monoamine neurotransmitters, as well as on genes involved in the clearance of glutamate and GABA from the synaptic cleft, because some of these processes were observed to be altered in Per 2 mutant mice ${ }^{23,47}$. Special attention was given to astrocyte-specific genes (Supplemental table 2).

In the hypothalamus (HYP), GABA transporter 1 (Gat1/Slc6a1) mRNA was significantly decreased in GPer2 $\left(G C r e^{+} P e r 2^{f l f l}\right)$ compared to control (GCre Per2 $\left.2^{f l f l}\right)$ animals (Fig. 5A). In the nucleus accumbens (NAc), mRNA expression of GABA transporter 2 (Gat2/Slc6a13) and dopamine receptor D3 (Drd3) was increased in GPer2 $\left(G C r e^{+}\right.$Per2 $\left.2^{f l f l}\right)$ compared to control $\left(G C r e^{-}\right.$Per2 $\left.2^{f / f l}\right)$ mice (Fig. 5B, C). In contrast, Gat1/Slc6a1 and Gat3/Slc6a11 were unaltered (Supplemental table 1). Interestingly, genes coding for enzymes involved in monoamine synthesis, such as tyrosine hydroxylase (Th) and monoamine degradation, such as monoamine oxidases $A$ (Maoa) and $B(\mathrm{Maob})$, as well as catechol-O-methyltrasferase (Comt) were similar between the two genotypes in the NAc (Fig. 5D-G), while Per 2 mRNA was significantly reduced in GPer2 mice (Fig. 5H). These results suggest that Per2 in glial cells is involved in the regulation of GABA signaling, rather than the regulation of monoaminergic signaling. This was further underlined by our findings on the analysis of neurotransmitters. We found that glutamate 

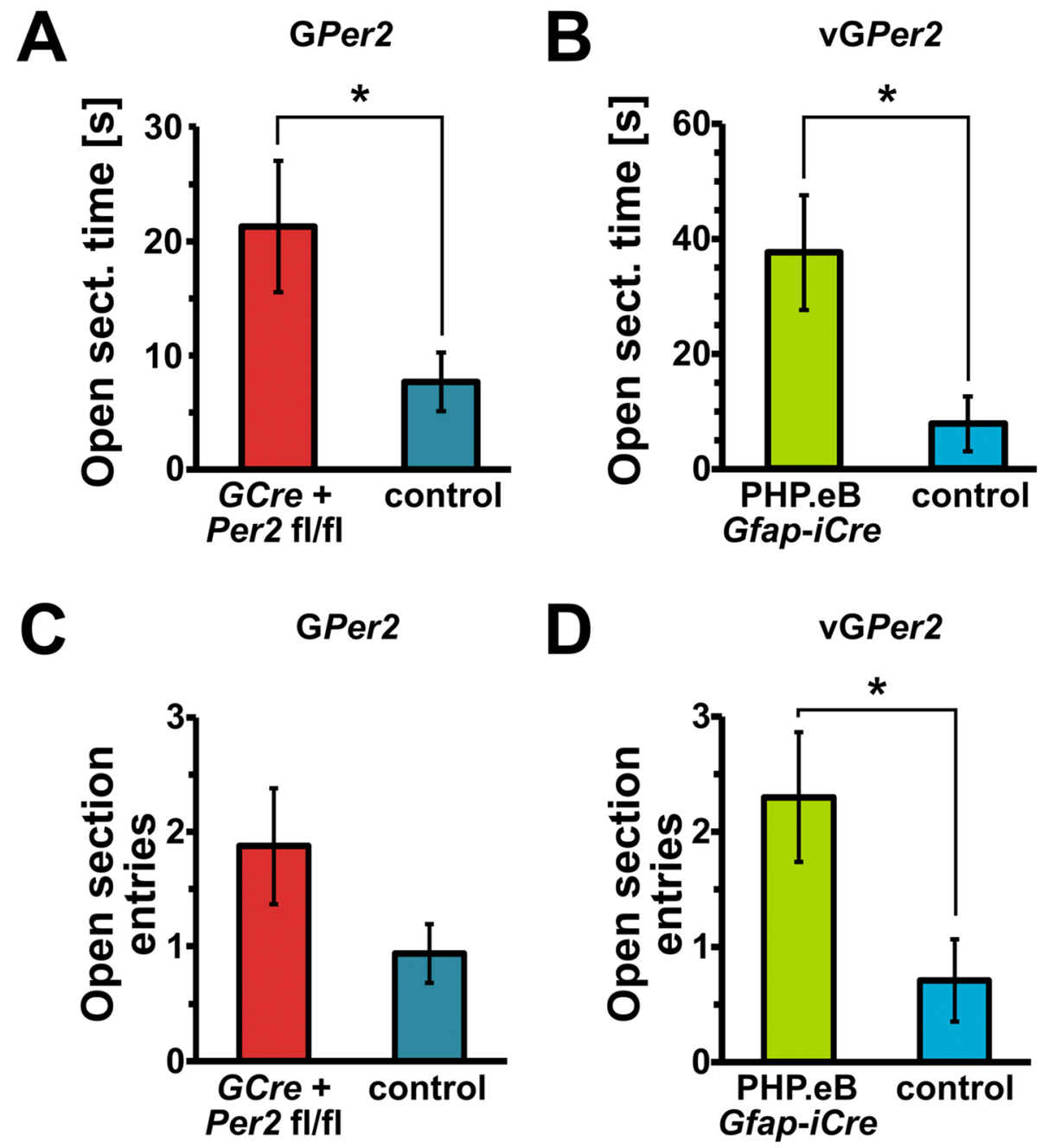

Figure 3. Reduced anxiety of GPer2 and vGPer2 animals in the elevated O-maze. (A) Time spent in the open area of the O-maze is longer in GPer2 $\left(G C r e^{+} P e r 2^{f / f l}\right.$, red $)$ animals compared to controls (blue) $(\mathrm{n}=16$ and 18 , respectively, two-tailed t-test, $\left.{ }^{\star} P<0.05\right)$. (B) Time spent in the open area of the $\mathrm{O}$-maze is significantly longer in vGPer2 (AAV-PHP.eB Gfap-iCre, green) compared to controls (blue) ( $\mathrm{n}=6$ and 7, respectively, two-tailed t-test, $\left.{ }^{\star} P<0.05\right)$. (C) Entries into the open section of the $\mathrm{O}$-maze are not significantly different between the GPer2 animals (red) and their controls (blue) ( $\mathrm{n}=16$ and 18 , respectively, two-tailed t-test, $P=0.15$ ). (D) The number of entries into the open section of the O-maze is higher in vGPer2 animals (green) compared to their controls (blue) ( $\mathrm{n}=6$ and 7 , respectively, two-tailed t-test, $\left.{ }^{\star} P<0.05\right)$.

(Glu) levels were significantly decreased in the NAc of GPer2 $\left(G C r e^{+} P e r 2^{f l f l}\right)$ mice (Fig. 5I). This difference, however, was not significant in the other brain areas investigated (dorsal striatum (DS), medial prefrontal cortex (mPFC), Supplemental table 3). We did also not observe changes in GABA and glutamine in the NAc, DS and mPFC (Supplemental table 3).

Taken together, our molecular studies examining 4 regions of interest suggest that lack of Per2 in glia affects mostly the NAc, and there the signaling pathways involving GABA and glutamate, as well as signaling via DRD3. NAc is therefore likely one of the regions involved in the observed behaviour.

Deletion of Per2 in glial cells of the NAc is sufficient to elicit a reduced despair phenotype. Since we observed most of the molecular changes in the NAc of GPer 2 animals, we wondered whether deletion of Per2 in glial cells of the NAc alone could elicit the phenotypes described above. Therefore, we injected our viral vectors directly into the NAc of Per $2^{f / f l}$ mice (Fig. 6A) using a stereotactic injection apparatus and termed the animals GNAcPer2. We saw that both AAV vectors, the PHP.eB Gfap-iCre as well as the AAV9 Gfap$i$ Cre significantly reduced immobility time in the FST (Fig. 6B, C). The results were comparable to the observations in GPer 2 and the vGPer2 animals, in which Per 2 was deleted in glial cells throughout the brain (Fig. 1 and 2). In contrast, we did not observe an effect on the time spent in the open section of the O-maze when AAV9 Gfap-iCre was injected into the NAc (Fig. 6D), suggesting that glial Per2 in the NAc was not involved in the regulation of anxiety-related behavior. 
A

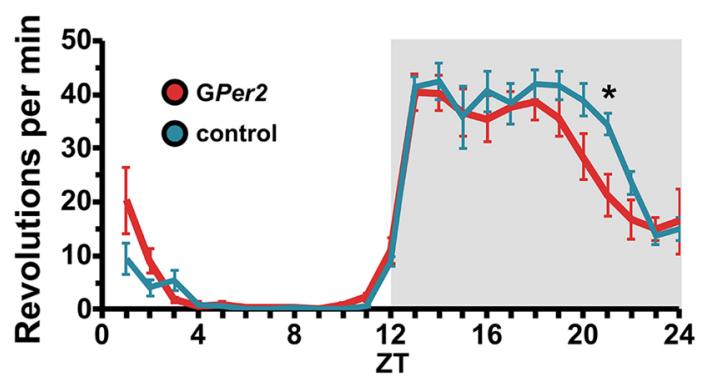

B

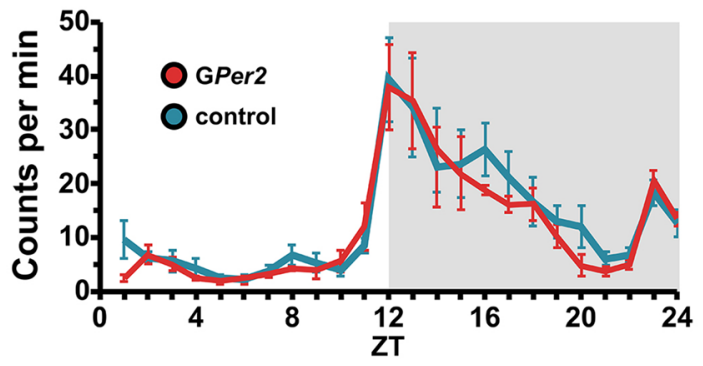

$\mathbf{E}$

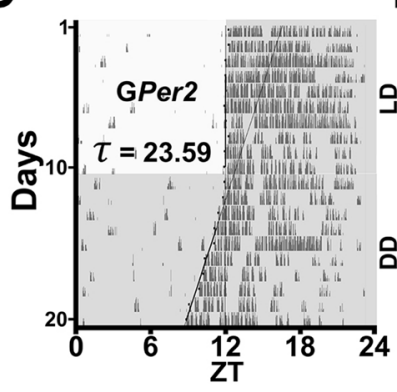

$\mathbf{F}$
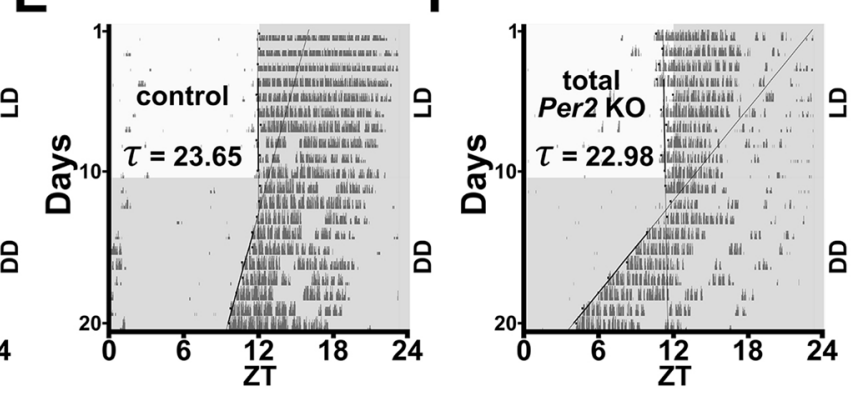

H

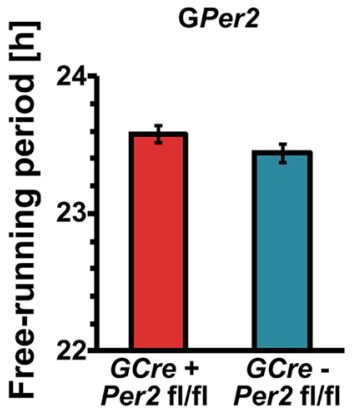

G

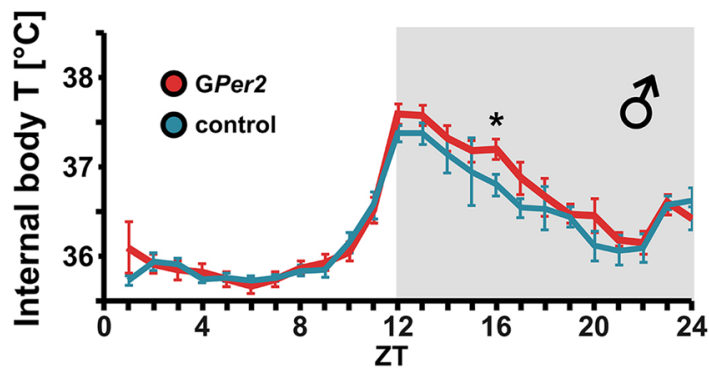

Figure 4. GPer2 mice have a normal circadian clock. (A) Wheel-running activity profile of GPer2 $\left(G C r e^{+} P e r 2^{f / f l}\right.$, red) and control $\left(G C r e-P e r 2^{f / f l}\right.$, blue) mice. The profiles are almost identical with a slight reduction of activity in GPer2 animals at zeitgeber time (ZT) 21. (B) General activity pattern of GPer2 (red) and control (blue) mice. The two profiles are not significantly different. (C) Circadian period of GPer2 (red) and control (blue) mice. No significant difference was observed between the two genotypes $(\mathrm{n}=6$, two-tailed t-test, $P>0.05$ ). (D) Representative wheel-running actogram of a GPer2 animal. Black vertical marks represent activity in the wheel. Upper part shows the activity under an LD 12:12 cycle. Lower part shows the activity under constant darkness conditions (DD). (E) Representative wheel-running actogram of a control animal. (F) Representative wheel-running actogram of a whole-body total Per2 knock-out animal. (G) Body temperature profile of male GPer2 (red) and control (blue) mice under LD conditions $(n=6)$. $(\mathbf{H})$ Body temperature profile of female GPer2 (red) and control (blue) mice under LD conditions $(n=6)$.

Taken together, our data provide evidence that Per2 expression in glial cells of the NAc is responsible for the regulation of despair-based behavior.

The phenotype of GPer2 mice is not the result of a defective glial molecular clock. To answer the question whether the observed phenotype of GPer 2 mice comes from a defect of the molecular clock in glia, we used the aforementioned viral approach to generate mice lacking glial Bmal1 (vGBmal1), an essential molecular clock gene (Fig. 7A, full size gel Suppl. Figure 1B). These mice showed no changes of immobility time in the FST (Fig. 7B) and no difference in time spent in the open section of the O-maze (Fig. 7C). Gat2/Slc6a13 was not significantly decreased, while $\operatorname{Drd} 3$ expression was significantly lower in the NAc (Fig. 7D-E). The behaviour of vGBmal1 animals shows that the phenotype observed in GPer 2 mice is not a direct result of a defect of the molecular clock, but rather a change in the output of the clock involving the Per2 gene. This is probably related to the function of the PER2 protein as a nuclear receptor co-regulator ${ }^{44}$.

To test whether the observed reduced despair and anxiety are specific to glial Per 2 function, we produced mice lacking Per 2 in neurons by utilizing the PHP.eB vector for genetic manipulation in adult animals. We systemically 
A

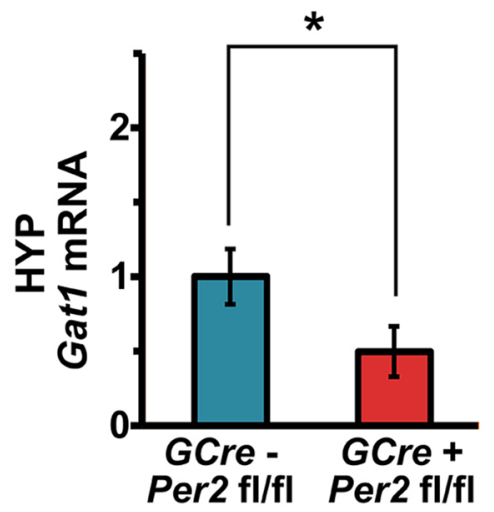

B
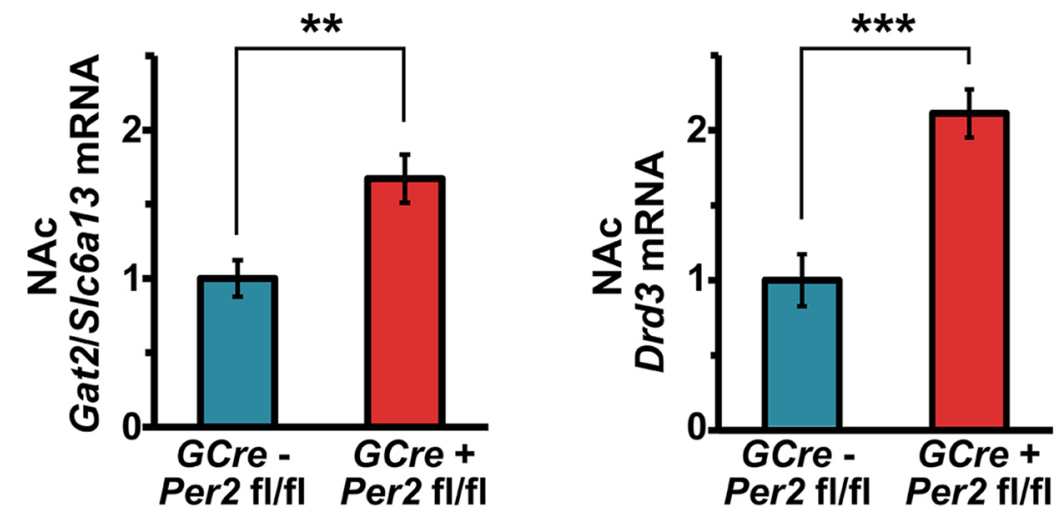

D

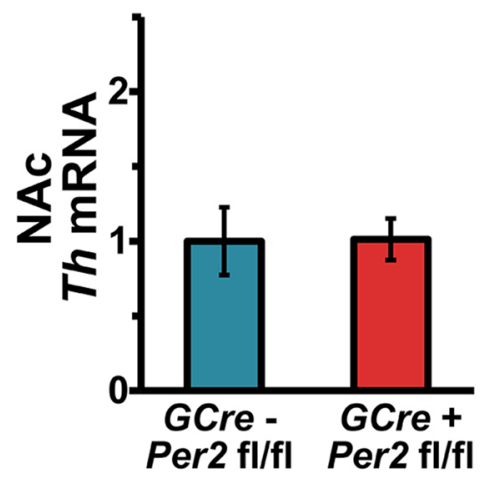

E

$\mathbf{F}$
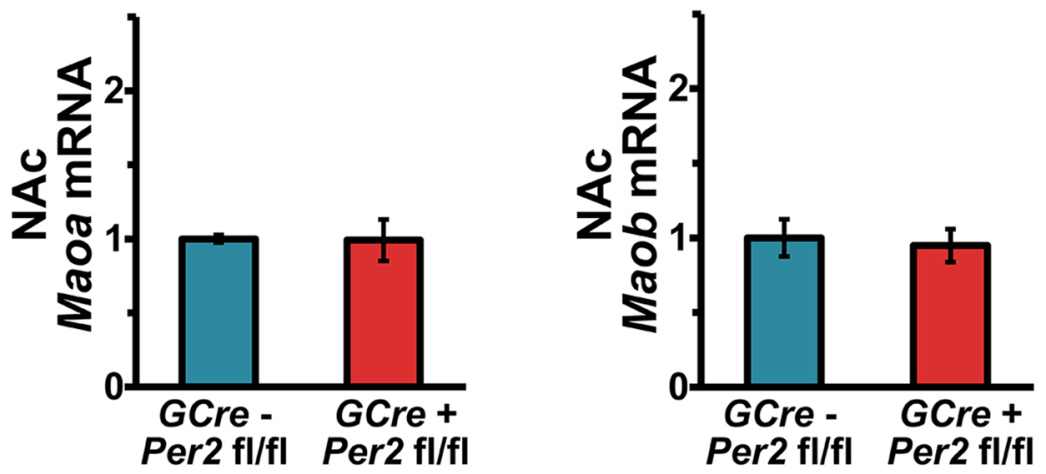

G

H
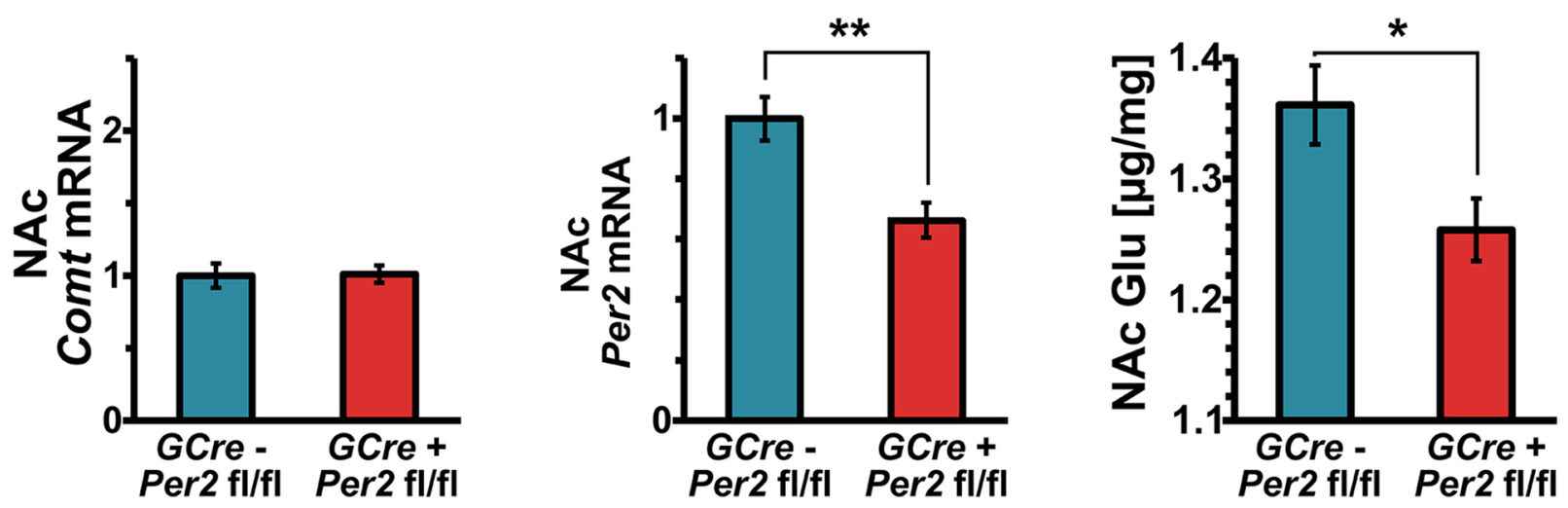

Figure 5. Molecular changes in GPer2 mice. (A) Expression of GABA transporter 1 (Gat1) mRNA in the hypothalamus (HYP) of GPer2 (GCre ${ }^{+} P e r 2^{f l / f l}$, red) control (GCre- Per2 $2^{f / f l}$, blue) at ZT2 (n=3, two-tailed t-test, $\left.{ }^{\star} P<0.05\right)$. (B) Expression of GABA transporter 2 (Gat2/Slc6a13) mRNA in the nucleus accumbens (NAc) of GPer2 (red) and control (blue) animals ( $\mathrm{n}=6$, two-tailed t-test, $\left.{ }^{\star \star} P<0.01\right)$. (C) Expression of dopamine receptor D3 (Drd3) mRNA in the NAc of GPer2 (red) and control (blue) animals ( $\mathrm{n}=6$, two-tailed t-test, $\left.{ }^{* * *} P<0.001\right)$. (D) Expression of tyrosine hydroxylase (Th) mRNA in the NAc of GPer2 (red) and control (blue) animals ( $\mathrm{n}=6$, two-tailed t-test, $P>0.05$ ). (E) Expression of monoamine oxidase A (Maoa) mRNA in the NAc of GPer2 (red) and control (blue) animals ( $\mathrm{n}=6$, two-tailed t-test, $P>0.05)$. (F) Expression of Maob mRNA in the NAc of GPer 2 (red) and control (blue) animals $(\mathrm{n}=6$, two-tailed t-test, $P>0.05)$. (G) Expression of catechol-O-methyltrasferase (Comt) mRNA in the NAc of GPer 2 (red) and control (blue) animals ( $\mathrm{n}=6$, two-tailed t-test, $P>0.05)$. $(\mathbf{H})$ Reduction of Per2 in GPer2 mice (red) compared to controls (blue). (I) Amount of glutamate (Glu) in the NAc of GPer2 (red) and control (blue) animals at ZT6 $\left(\mathrm{n}=5\right.$, two-tailed t-test, $\left.{ }^{\star} P<0.05\right)$. 

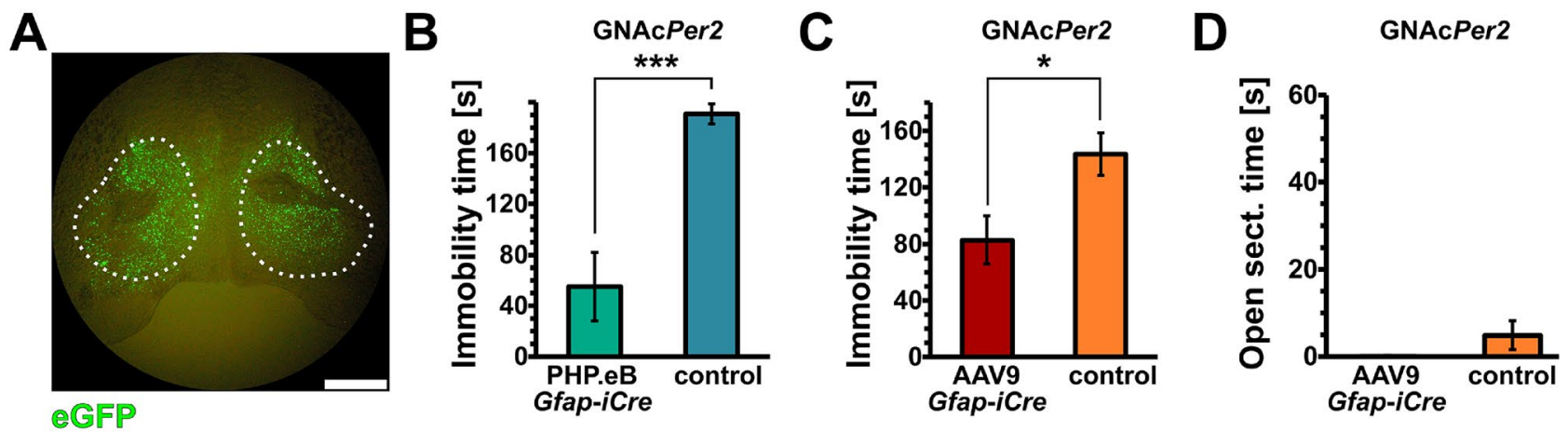

Figure 6. Deletion of Per2 in glia of the NAc evokes reduced despair but has no effect on anxiety-related behavior. (A) Injection of the AAVs into the NAc as revealed by the eGFP reporter fluorescence. White dotted area delineates the NAc. Scale bar: $1 \mathrm{~mm}$. (B) Immobility time in the forced swim test (FST) of PHP.eB Gfap$i C r e$ (green) and non-injected control (Per2 $2^{f l f l}$, blue) animals is shown $(\mathrm{n}=4$ or 5 , respectively, two-tailed $\mathrm{t}$-test, $\left.{ }_{* * *} P<0.001\right)$. (C) FST of AAV9 Gfap-iCre Per $2^{f / f l}$ (red) and Gfap-iCre Per2 ${ }^{\mathrm{WT}}$ littermate control (orange) NAc injected mice is shown $\left(\mathrm{n}=7\right.$ or 8 , two-tailed t-test, $\left.{ }^{\star} P<0.05\right)$. Deletion of glial Per 2 in the NAc with both types of AAV capsid, but same expression cassette, appears to be sufficient to evoke reduced despair. (D) Time spent in the open area of the O-maze is similar in both NAc injected AAV9 Gfap-iCre and AAV9 control animals, suggesting no effect of Per 2 glial deletion in the NAc on anxiety-related behavior ( $\mathrm{n}=7$ or 8 , two-tailed $\mathrm{t}$-test, $P>0.05)$.

delivered a construct in which with the Synapsin 1 (Syn1) promoter drove expression of $i$ Cre in neurons of Per $2^{f l / l}$ mice, and we named these mice vNPer2 (Fig. 7F, full size gel Suppl. Figure 1C).

To our surprise, these mice also exhibited a lowered immobility time in the FST (Fig. 7G), albeit to a smaller degree than vGPer2 mice (Fig. 2). However, we did not observe any change in the O-maze test (Fig. 7H). Interestingly, these mice also showed an increase of Gat2/Slc6a13, but normal expression of Drd3 in the NAc (Fig. 7I-J). Syn1-driven neuronal Per 2 knock-out in adult animals was also associated with low activity levels under LD conditions (Fig. 7K). Interestingly, some mice showed difficulties in entrainment to the LD light cycle (Fig. 7L) compared to normal activity patterns of mice injected with an equivalent virus lacking $i$ Cre (Fig. $7 \mathrm{M}$ ). This was an observation that we did not expect, as cross-bred neuronal Per2 KO mice (Nestin-driven Cre) show normal activity patterns under LD conditions ${ }^{13}$.

\section{Discussion}

Mood-related behaviors including depression are determined by several and sometimes synergistic factors illustrating the multifactorial nature of these neurological disturbances. In addition to the molecular complexity of these processes, cellular heterogeneity and topological organization complicate the understanding of the functioning of the brain even further. Previous studies have shown that at the molecular level genes involved in the regulation of the circadian clock can affect mood-related behaviors in mice ${ }^{15,23,34}$ and most likely also in humans ${ }^{28,38}$. Anatomical and genetic tracing studies have also revealed particular brain regions to be involved in various aspects of mood regulation.

Our previous studies have implicated a role of the clock gene Per2 in mood-related behavior involving the mesolimbic dopaminergic system ${ }^{23}$. However, the contribution of glial cells in this process is poorly understood ${ }^{52}$. Therefore, we were interested to investigate the role of glial Per2. We used three approaches to investigate this question. First, we used genetic tools to delete Per2 specifically in glial cells, including astrocytes (Fig. 1). Second, we deleted Per 2 in glial cells of the adult animal via systemic application of an engineered AAV that could pass the BBB (Fig. 2). This experiment was performed to exclude potential developmental effects that could contribute to behavioral alterations in the genetic approach. Third, we deleted Per2 specifically in glial cells of the NAc by injection of AAVs expressing Cre recombinase. All three approaches, the genetic as well as the AAV approaches revealed that absence of Per 2 in glial cells, in particular the NAc, reduced the immobility time of mice in the FST, a test that assesses despair-based behavior, one of the aspects of depression. Glial deletion of Per2 resulted in more active swimming in the FST, correlating with a manic behavior as we have previously observed in wholebody Per 2 mutant mice ${ }^{23}$. Interestingly, pharmacologic glial ablation in the prefrontal cortex has been reported to increase immobility in the FST and as a consequence made the animals more depressive $\mathrm{s}^{5}$ This phenotype is the opposite from what we observed in our GPer2 and vGPer2 mice and indicates that in our animal models glial cells are still functional and not eliminated. Hence, our observations are specific to glial Per 2 function and are very unlikely related to processes leading to glial cell death.

The processes affected by lack of Per 2 in glial cells are most likely not related to the circadian clock mechanism, because GPer 2 animals display no abnormalities in clock parameters such as period or activity distribution and body temperature fluctuations (Fig. 4). If the disturbance of the clock mechanism in astrocytes would play a major role, a desynchronization between astrocytes and neurons would be expected, which would ultimately lead to arrhythmic activity of mice under constant darkness conditions. This is, however, not what we observed (Fig. 4). Furthermore, deletion of Bmal1 in glial cells did not affect immobility time in the FST (Fig. 7), suggesting that the clock itself does not cause the phenotype we observed in glial Per2 knock-out animals. Therefore, we 

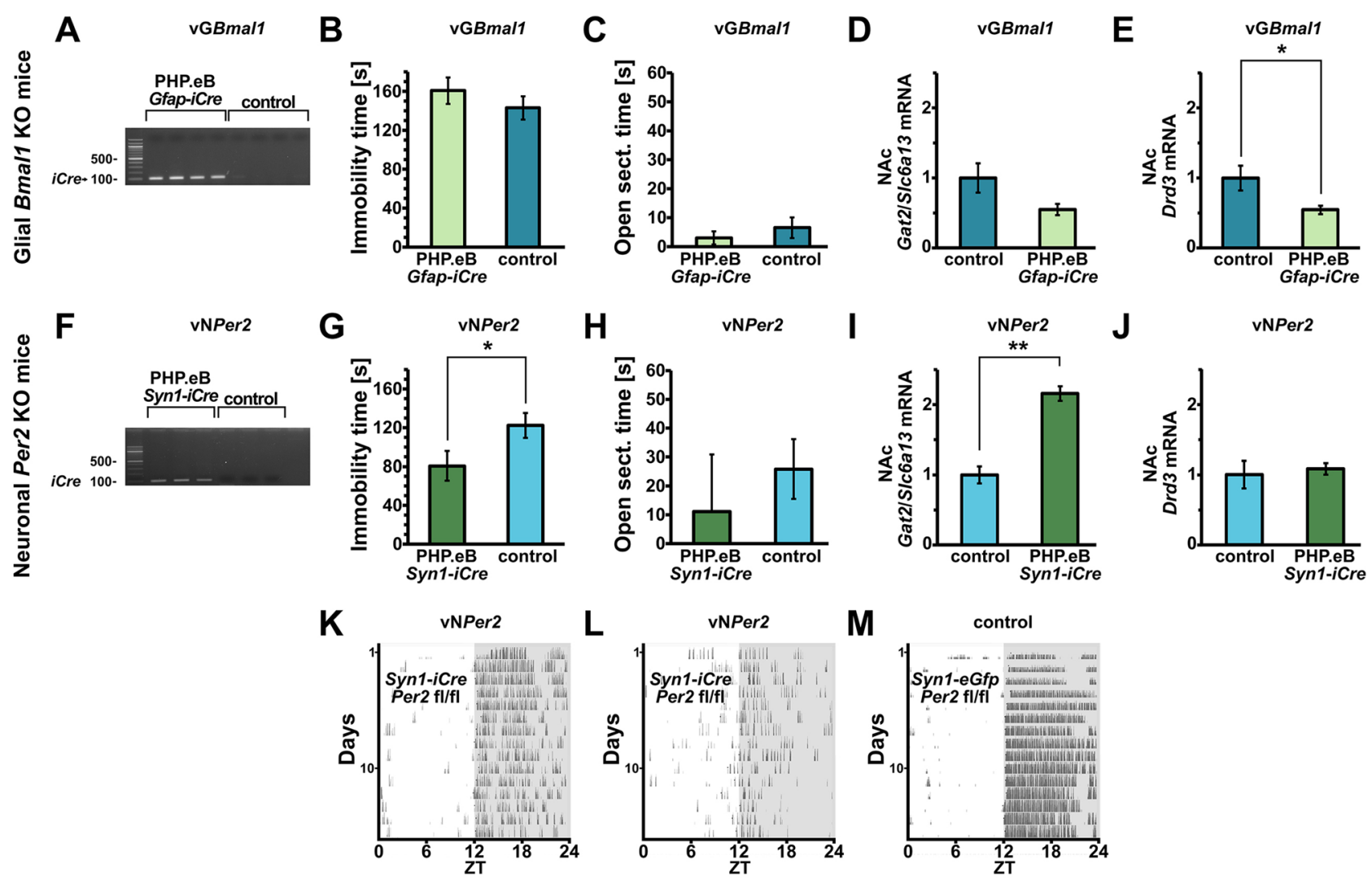

Figure 7. Deletion of Bmal1 in glia does not influence despair- or anxiety-related behavior, while neuronal Per2 deletion influences despair. (A) Agarose gel showing the expression of intravenously viral-delivered $i$ Cre in total NAc tissue of Bmalf ${ }^{f l / f l}$ and control animals $(n=4)$. All probes and markers were loaded on the same gel and the resulting photograph was not separated and recomposed. (B) Glial Bmal1 $\mathrm{KO}$ mice do not show a difference in the FST $(n=8)$ or $(\mathbf{C})$ O-maze $(n=8)$. Their NAc expression of $(\mathbf{D})$ Gat2 is not significantly reduced, whereas (E) $\operatorname{Drd} 3$ expression is significantly lower in the KO group $\left(\mathrm{n}=7\right.$ or 8 , two-tailed $\mathrm{t}$-test, $\left.{ }^{\star} P<0.05\right)$. (F) Agarose gel showing the presence of intravenously viral-delivered $i$ Cre in total NAc tissue of Per $2^{f / f l}$ and control animals $(n=3)$. All probes and markers were loaded on the same gel and the resulting photograph was not separated and recomposed. In comparison to vGBmal1, vNPer2 mice have $(\mathbf{G})$ a reduced immobility time in the FST $\left(\mathrm{n}=11\right.$ and 12, respectively, two-tailed t-test, $\left.{ }^{\star} P<0.05\right)$, but $(\mathbf{H})$ show no difference to controls injected with an equivalent virus, but lacking $i C r e$, in the $\mathrm{O}$-maze $(\mathrm{n}=11$ and 12 , respectively). (I) Their Gat2/Slc6a13 expression is upregulated $\left(\mathrm{n}=3\right.$, two-tailed t-test, $\left.{ }^{\star *} P<0.01\right)$, while $(\mathrm{J})$ the $\operatorname{Drd} 3$ levels are unaltered in the NAc $(\mathrm{n}=3)$. $(\mathbf{K})$ A representative vNPer 2 mouse shows reduced locomotor activity in a wheel-running cage, as represented with an actogram, where each line represents a day of activity, the latter illustrated with the height and frequency of vertical lines. (L) Some KO mice also showed difficulties entraining to the light cycle. (M) The mice injected with the control virus, which lacked $i C r e$, showed normal locomotor activity.

hypothesize that the phenotypes we describe here are probably related to PER2 output functions, e.g. as nuclear receptor co-regulator ${ }^{4}$. PER2 may potentially act as a nuclear receptor co-regulator, which would correlate with the altered levels of Gat2/Slc6a13 and Drd3 mRNA that we observed in the NAc (Fig. 5B). GABA transporters as well as $\operatorname{Drd} 3$ have been implicated in mood-related behavior. $\operatorname{Drd} 3$ has been shown to be regulated by the transactivator RORa and repressor REV-ERBa ${ }^{25}$, both of which bind to the same promoter elements (ROREs) and of which REV-ERBa is modulated by PER2 ${ }^{44}$. However, the regulation of Gat $2 / S l c 6 a 13$ remains elusive.

Since depression is not only related to despair, we also tested our animals in the elevated O-maze. This test addresses anxiety-related behavior, which is an important component of depression. We observed that both GPer2 as well as vGPer 2 mice were less anxious, because they spent more time in the open area of the O-maze (Fig. 3). Interestingly, however, the animals with Per2 deleted in the glia of the NAc only (GNAcPer2) did not show this phenotype (Fig. 6D). From this we conclude that Per2 in glia of the NAc may not be important for anxiety-related behavior and specifically affects despair. Hence, glial Per2 in other brain regions, such as the amygdala $^{17}$, the bed nucleus of the stria terminalis (BNST) ${ }^{41}$ or the subgenual anterior cingulate cortex $(\operatorname{sgACC})^{3}$, part of the medial prefrontal cortex (mPFC), may be involved in the modulation of anxiety. Interestingly, vNPer 2 mice showed no difference in anxiety perception (Fig. $7 \mathrm{H})$ and it seems that the reduced anxiety is mediated mainly through glial Per2. This suggests that distinct brain regions and also different underlying mechanisms lead to reduced despair and anxiety in GPer2 and vGPer 2 mice. Whereas the vNPer2 mice also showed increased Gat2 levels, as observed in GPer2 mice, they had no alterations in Drd3 expression. 
We did not test anhedonia, another aspect of depression, because whole-body Per2 mutant mice did not show any changes in the sucrose preference test ${ }^{47}$. Therefore, we assumed that glial Per2 plays a subordinate or no role in the anhedonia aspect of depression.

Our previous studies describing whole-body Per2 mutant mice highlighted a functional involvement of Per2 in the regulation of monoamine degradation ${ }^{23}$. In this study, we find that glial Per2 appears to play a role in the regulation of glutamate, GABA and dopaminergic signaling, but not monoamine metabolism (Fig. 5), at least not at the observed time-point. There is ample evidence that both, monoaminergic and GABAergic signaling regulate depressive disorders ${ }^{31}$, and Per2 might be involved in both. If this hypothesis was correct, then we would expect that deletion of Per 2 in neurons would still affect mood-related behaviors. Our results are consistent with this view, because vNPer 2 mice show reduced immobility in the FST (Fig. 7F).

Alterations of glial function are considered to modify glutamate reuptake $e^{14}$, which may be the reason why we observed reduced glutamate levels in the NAc of our GPer 2 knock-out animals (Fig. 5I). Interestingly, however, we did not observe changes in expression of glutamate transporters Eaat1 and Eaat2 (Supplemental table 2). This is in contrast with our previous study investigating whole-body Per2 mutant mice, which show decreased Eaat 1 levels ${ }^{47}$. Hence, it appears that lack of Per2 function in neurons and astrocytes leads to changes in Eaat 1 expression, illustrating the complex interplay between neurons and astrocytes in the regulation of glutamate signaling and mood-related behavior.

Overall, our results provide evidence for a specific role of glial Per2 in mood-related behavior, accompanied by dysregulation of components of the glutamatergic, GABAergic and dopaminergic signaling pathways.

\section{Materials and methods}

All experiments were approved by the Bundesamt für Umwelt BAFU and the State Veterinarian of the Canton of Fribourg.

Animals and housing. All mice were housed with food and water ad libitum in transparent plastic cages (267 mm long $\times 207 \mathrm{~mm}$ wide $\times 140 \mathrm{~mm}$ high; Techniplast Makrolon type 2 1264C001) with a stainless-steel wire lid (Techniplast 1264C116), kept in light- and soundproof ventilated chambers. All mice were entrained to a LD 12:12 cycle, and the time of day was expressed as zeitgeber time (ZT; ZT0 lights on, ZT12 lights off). Twoto four-month-old males and females were used for the experiments unless otherwise stated. Males were used for the FST, while females were used for the O-maze and mixed genders to monitor activity. Housing as well as experimental procedures were performed in accordance with the guidelines of the Schweizer Tierschutzgesetz and the Declaration of Helsinki. The state veterinarian of the Canton of Fribourg approved the protocols. The study was carried out in compliance with the ARRIVE guidelines.

Conditional glial Per2 knock-out animals were generated using Gfap-Cre mice (Jackson lab FVB-Tg(GFAPcre) $25 \mathrm{Mes} / \mathrm{J}$, stock no. 004600 , created by the laboratory of A. Messing) that were cross-bred with our Per2 floxed animals $\left({ }^{13}\right.$, European Mouse Mutant Archive (EMMA) strain ID EM: 10,599, B6,129P2-Per2 ${ }^{\text {tm1Ual }} /$ Biat). The resulting GPer2 line was back-crossed to the C57BL/6 strain. The Per2 ${ }^{\mathrm{Brdm} 1}$ mutant mice ${ }^{51}$ and their wild-type littermate controls were on a mixed 129 and C57BL/6 background.

Viruses used and their application. The following viruses, produced by the Viral Vector Facility of the Neuroscience Center Zurich, were used: v95-PHP.eB (ssAAV-PHP.eB/2-hGFAP-EGFP-WPRE-hGHp(A)) as the control virus and v232-PHP.eB (ssAAV-PHP.eB/2-hGFAP-EGFP_iCre-WPRE-hGHp(A)) as the virus for recombination, v344-9 (ssAAV-9/2-shortCAG-chI[1 $\times(\mathrm{shm} / \mathrm{rNS})]-$ EGFP-WPRE-SV40p(A)) and v25-PHP. eB (ssAAV-PHP.eB/2-CAG-EGFP_Cre-WPRE-SV40p(A)) as controls for BBB permeability determination, while stereotactic injections were also performed with constructs v95 and v232 in the AAV9 capsid. All viruses expressed the green fluorescent protein as a marker.

For intravenous delivery of viruses, mice were sedated and chemically restrained with an intraperitoneal injection of $40 \mathrm{mg}$ per $\mathrm{kg}$ body mass ketamine and $0.15 \mathrm{mg}$ per $\mathrm{kg}$ medetomidine in saline and placed on a heating pad, while hydrogel was applied to their eyes. We injected $10^{11}$ viral genomes per mouse in a total volume of $200 \mu \mathrm{l}$ PBS via the lateral tail vein. The sedation was reversed with atipamezole in 5 times the dose of medetomidine.

Whole-brain fluorescent imaging was performed using the IVIS Lumina II (Caliper LifeSciences) and the accompanying Living Image software (version 4.2.0.14335). Settings: excitation $465 \mathrm{~nm}$, filter GFP, exposure time $1 \mathrm{~s}$, light level low, binning low/small.

Forced swim test (FST). The Porsolt's forced swim test has been described in detail elsewhere ${ }^{23,39}$. Briefly, the mice were placed into a cylinder filled with water, where they were left for $6 \mathrm{~min}$. The first $2 \mathrm{~min}$ were discarded as adaptation period, while the following $4 \mathrm{~min}$ were scored. The test was performed for four consecutive days. The first day is regarded as adaptation and was discarded. The following three days were manually scored for immobility time - the amount of time a mouse passively floated during the 4 min test window. An average immobility time for each mouse was calculated and then these were pooled according to the assigned experimental group. In the case of animals injected with the PHP.eB virus, the forced swim test was performed 4 weeks post injections ${ }^{10}$. All forced swim tests were performed at ZT $6,6 \mathrm{~h}$ after lights were switched on in a $12 \mathrm{~h} \mathrm{light,}$ $12 \mathrm{~h}$ dark environment ${ }^{23}$. The water temperature was always adjusted to $26+1-1^{\circ} \mathrm{C}$.

The FSTs were recorded from the side view. The automated forced swim test analysis was performed with a custom written program that evaluated the relative pixel difference in a rectangular area spanning the width of the swim tank and having the height equal to one sixth of the width, measured from the water surface towards the bottom of the tank. Each pixel in the test area was evaluated for changes between frames in red, green and 
blue on the decimal scale, the differences were averaged and divided by the test area, resulting in a graph that showed relative pixel change per area over time.

O-maze. The anxiety-based O-maze test was performed on an elevated $5.5 \mathrm{~cm}$ wide circular runway with an outer diameter of $46 \mathrm{~cm}$, which was divided into 4 sections, with 2 opposing closed sections. The time spent in the open sections was evaluated based on video recording. Entry into the open section was considered when the mouse entered it with all 4 paws. To avoid habituation to the test environment, a single test of 5 min was performed. Two mice with values more than 2 standard deviations from the group mean were excluded from the study as outliers: one mouse was removed from the GPer 2 group (276 s or $92 \%$ of the time in the open section) and one mouse from the corresponding control group (40 s in the open section). One mouse was also excluded from the vGPer 2 group ( $274 \mathrm{~s}$ or $91 \%$ of the time in the open section).

Tissue isolation, gene expression analysis and neurotransmitter quantification. For both gene expression analysis and neurotransmitter quantification, fresh brain tissue was dissected and immediately submerged into liquid nitrogen. For the list of primers, please see Supplemental table 4. For gene expression analysis, RNA was isolated using the Macherey-Nagel RNA Plus kit and reverse transcribed using the Invitrogen SuperScript II. qPCRs were performed using the RotorGene 6000, with the KAPA Probe or KAPA SYBR master mix reagent.

Surgical procedures. The surgical procedures were performed as previously described ${ }^{33}$. Briefly, mice were anesthetized with $80 \mathrm{mg}$ per $\mathrm{kg}$ ketamine and $0.30 \mathrm{mg}$ per $\mathrm{kg}$ medetomidine, while the anesthesia was reversed by atipamezole in 5 times the dose of medetomidine. Before surgical procedures, the depth of anesthesia was checked by an absence of a reflex when pinching the skin between the toes of the mouse 5-10 min after application of the anesthetic. In case of a reflex, a quarter of the initial dose of the anesthetic was additionally administered. For measurements of internal body temperature and general activity, a wireless biochip (VitalView system) was implanted into the abdominal cavity (Starrlife Sciences, VitalView Data Acquisition System, Instruction Manual, Software Version 5.1). Stereotactic injections were performed with a pulled glass pipette, which allowed injections of $2 \times 200 \mathrm{nl}$ of the virus bilaterally into the nucleus accumbens (NAc) with stereotactic coordinates of 1.60 anterior-posterior, $+/-1.00$ medio-lateral and 4.50 dorso-ventral. Surgical procedures were followed by administration of carprofen at $10 \mathrm{mg}$ per $\mathrm{kg}$.

Immunohistochemistry. Brains were harvested after mice were cardiovascularly perfused with saline and 4\% PFA, and the tissue was left in PFA over night, and then transferred to $30 \%$ sucrose in PBS for two days for cryoprotection. $40 \mu \mathrm{m}$ sections were cut using a cryostat and kept at $-20{ }^{\circ} \mathrm{C}$ in an antigen preservation solution $(1 \% \mathrm{~m} / \mathrm{m}$ polyvinyl pyrrolidone in a $1: 1$ mixture of PBS and ethylene glycol) until subsequent steps of washing, blocking with permeabilization, antigen retrieval and antibody staining (anti-PER2, Alpha Diagnostic, cat. PER21-A, 1 : 200 dilution; anti-GFAP, Abcam, cat. ab53554, $1: 500$ dilution; anti-NeuN, Merck Millipore, cat. mab377, $1: 250$ dilution). The procedures have been described by our laboratory in detail elsewhere ${ }^{9}$. Images were acquired using a Leica SP5 confocal microscope.

Wheel-running experiments, general activity and internal body temperature measurements. Wheel-running experiments were performed using custom built cages, according to local legislation on animal experimentation, which had a stainless-steel wire running wheel with a diameter of $11.5 \mathrm{~cm}$. On the axis, a system with a magnet closed a switch for each wheel revolution. The data was digitalized using an interface from Actimetrics and the activity was recorded and processed using the ClockLab software version 6.0.54.

This allowed acquisition of activity patterns in LD conditions, as well as monitoring of the subjective day length under constant conditions. For this, the mice were entrained to LD conditions and then released into total darkness for a minimum of 10 days. The first three days were discarded as assimilation, while the subsequent 7 days were evaluated for a shift of activity onset on each consecutive day in order to give a measure of the length of the subjective day or free-running period, as previously described ${ }^{27,33}$.

Telemetrics was performed with the VitalView system, which is used for wireless measurements of general mouse activity, as well as internal body temperature, allowing monitoring of behavioural and physiological changes in free-running animals. For the biochip implantation, please see 'Surgical procedures'. All the procedures have been described in detail elsewhere ${ }^{33}$.

Flow cytometry. At the desired time-point, brain tissue was harvested, dissected, stored in a test tube and submerged into cooled isopentane. The samples, still in the isopentane bath, were then stored at $-80^{\circ} \mathrm{C}^{43}$. For flow cytometry, the samples were diced with razor blades and digested under heat and agitation with the Neural Tissue Dissociation Kit (T) (130-093-231) from Miltenyi Biotech according to the manufacturer's protocol. Single-cell suspensions were obtained by straining through a $70 \mu \mathrm{m}$ mesh filter, after which the strained cells were washed twice in FACS buffer (PBS with 5\% fetal calf serum and $5 \mathrm{mM}$ EDTA). Then, myelin debris were removed using the Myelin Removal Beads II kit (130-096-731) from Miltenyi Biotech. Myelin-depleted cell suspensions were incubated with an anti-CD16/CD32 Fc-blocking antibody (BD Biosciences) for 30 min at $4{ }^{\circ} \mathrm{C}$ to avoid Fc receptor binding and then washed once with FACS buffer. The cells were stained with fluorophoreconjugated antibodies and analyzed on a MACSQuant flow cytometer (Miltenyi Biotech) or sorted using the FACSAria Fusion cell sorter (BD Biosciences). Flow cytometry analysis was based on viable and single-cell gaiting strategies, as previously described ${ }^{45}$. Antibodies targeted CD11b (fluorophore PECy7, ref. 552,850, BD 
Pharmingen), CD90.2 (fluorophore PE, ref., 130-102-489, Miltenyi Biotech) and GLT1 (fluorophore ATTO 633, ref., AGC-022-FR, Alomone lab), and were used in a 1:100 dilution.

Received: 19 January 2021; Accepted: 31 May 2021

Published online: 10 June 2021

\section{References}

1. Abarca, C., Albrecht, U. \& Spanagel, R. Cocaine sensitization and reward are under the influence of circadian genes and rhythm. Proc. Natl. Acad. Sci. U S A 99, 9026-9030 (2002).

2. Albrecht, U. \& Ripperger, J. A. Circadian clocks and sleep: impact of rhythmic metabolism and waste clearance on the brain. Trends Neurosci. 41, 677-688 (2018)

3. Alexander, L. et al. Over-activation of primate subgenual cingulate cortex enhances the cardiovascular, behavioral and neural responses to threat. Nat. Commun. 11, 5386 (2020).

4. Altshuler, L. L. et al. Amygdala astrocyte reduction in subjects with major depressive disorder but not bipolar disorder. Bipolar. Disord. 12, 541-549 (2010).

5. Banasr, M. \& Duman, R. S. Glial loss in the prefrontal cortex is sufficient to induce depressive-like behaviors. Biol. Psychiatr. 64, 863-870 (2008).

6. Barca-Mayo, O. et al. Astrocyte deletion of Bmall alters daily locomotor activity and cognitive functions via GABA signalling. Nat. Commun. 8, 14336 (2017).

7. Bhagwagar, Z. et al. Low GABA concentrations in occipital cortex and anterior cingulate cortex in medication-free, recovered depressed patients. Int. J. Neuropsychopharmacol. 11, 255-260 (2008).

8. Brancaccio, M. et al. Cell-autonomous clock of astrocytes drives circadian behavior in mammals. Science 363, 187-192 (2019).

9. Brenna, A. et al. Cyclin-dependent kinase 5 (CDK5) regulates the circadian clock. Elife 8, 10 (2019).

10. Challis, R. C. et al. Systemic AAV vectors for widespread and targeted gene delivery in rodents. Nat. Protoc. 14, 379-414 (2019).

11. Chan, K. Y. et al. Engineered AAVs for efficient noninvasive gene delivery to the central and peripheral nervous systems. Nat. Neurosci. 20, 1172-1179 (2017).

12. Chappuis, S. et al. Role of the circadian clock gene Per2 in adaptation to cold temperature. Mol. Met. 2, 184-193 (2013).

13. Chavan, R. et al. Liver-derived ketone bodies are necessary for food anticipation. Nat. Commun. 7, 10580 (2016).

14. Choudary, P. V. et al. Altered cortical glutamatergic and GABAergic signal transmission with glial involvement in depression. Proc. Natl. Acad. Sci. U S A 102, 15653-15658 (2005).

15. Chung, S. et al. Impact of circadian nuclear receptor REV-ERBalpha on midbrain dopamine production and mood regulation. Cell 157, 858-868 (2014).

16. Cipriani, A. et al. Comparative efficacy and acceptability of 21 antidepressant drugs for the acute treatment of adults with major depressive disorder: a systematic review and network meta-analysis. Lancet 391, 1357-1366 (2018).

17. Davis, M. The role of the amygdala in fear and anxiety. Annu. Rev. Neurosci. 15, 353-375 (1992).

18. Dibner, C., Schibler, U. \& Albrecht, U. The mammalian circadian timing system: organization and coordination of central and peripheral clocks. Annu. Rev. Physiol. 72, 517-549 (2010).

19. Duncan, G. E., Johnson, K. B. \& Breese, G. R. Topographic patterns of brain activity in response to swim stress: assessment by 2-deoxyglucose uptake and expression of Fos-like immunoreactivity. J. Neurosci. 13, 3932-3943 (1993).

20. Etievant, A. et al. Astroglial control of the antidepressant-like effects of prefrontal cortex deep brain stimulation. EBioMedicine 2, 898-908 (2015).

21. Gadea, A. \& Lopez-Colome, A. M. Glial transporters for glutamate, glycine, and GABA: II. GABA transporters. J. Neurosci. Res. 63, 461-468 (2001).

22. GBD. Disease and Injury Incidence and Prevalence Collaborators (2018). Global, regional, and national incidence, prevalence, and years lived with disability for 354 diseases and injuries for 195 countries and territories, 1990-2017: a systematic analysis for the Global Burden of Disease Study 2017. Lancet 392, 1789-1858 (2017).

23. Hampp, G. et al. Regulation of monoamine oxidase A by circadian-clock components implies clock influence on mood. Curr. Biol. 18, 678-683 (2008).

24. Harno, E., Cottrell, E. C. \& White, A. Metabolic pitfalls of CNS Cre-based technology. Cell Metab. 18, 21-28 (2013).

25. Ikeda, E. et al. Molecular mechanism regulating 24-hour rhythm of dopamine D3 receptor expression in mouse ventral striatum. Mol. Pharmacol. 83, 959-967 (2013).

26. Jackson, F. R., You, S. \& Crowe, L. B. Regulation of rhythmic behaviors by astrocytes. Rev. Dev. Biol. 9, 372 (2020).

27. Jud, C., Schmutz, I., Hampp, G., Oster, H. \& Albrecht, U. A guideline for analyzing circadian wheel-running behavior in rodents under different lighting conditions. Biol. Proced. Online 7, 101-116 (2005).

28. Li, J. Z. et al. Circadian patterns of gene expression in the human brain and disruption in major depressive disorder. Proc. Natl. Acad. Sci. U S A 110, 9950-9955 (2013).

29. Lindhorst, A., Bechmann, I. \& Gericke, M. Unspecific DNA recombination in AdipoqCre-ER(T2)-mediated knockout approaches in transgenic mice is sex-, age- and genotype-dependent. Adipocyte 9, 1-6 (2020).

30. Luo, L. et al. Optimizing nervous system-specific gene targeting with cre driver lines: prevalence of germline recombination and influencing factors. Neuron 106, 37-65 (2020).

31. Luscher, B., Shen, Q. \& Sahir, N. The GABAergic deficit hypothesis of major depressive disorder. Mol. Psychiatr. 16, $383-406$ (2011).

32. Magistretti, P. J. Neuron-glia metabolic coupling and plasticity. J. Exp. Biol. 209, 2304-2311 (2006).

33. Martini, T., Ripperger, J. A. \& Albrecht, U. Measuring food anticipation in mice. Clocks Sleep 1, 65-74 (2019).

34. McClung, C. A. et al. Regulation of dopaminergic transmission and cocaine reward by the Clock gene. Proc. Natl. Acad. Sci. U S A 102, 9377-9381 (2005).

35. Miyazaki, J. et al. Expression vector system based on the chicken beta-actin promoter directs efficient production of interleukin-5. Gene 79, 269-277 (1989).

36. Nestler, E. J. \& Carlezon, W. A. Jr. The mesolimbic dopamine reward circuit in depression. Biol. Psychiatr. 59, 1151-1159 (2006).

37. Pariante, C. M. \& Lightman, S. L. The HPA axis in major depression: classical theories and new developments. Trends Neurosci. 31, 464-468 (2008).

38. Partonen, T. et al. Three circadian clock genes Per2, Arntl, and Npas2 contribute to winter depression. Annu. Med. 39, 229-238 (2007).

39. Porsolt, R. D., Anton, G., Blavet, N. \& Jalfre, M. Behavioural despair in rats: a new model sensitive to antidepressant treatments. Eur. J. Pharmacol. 47, 379-391 (1978).

40. Quintana, A. et al. Lack of GPR88 enhances medium spiny neuron activity and alters motor- and cue-dependent behaviors. Nat. Neurosci. 15, 1547-1555 (2012). 
41. Rodriguez-Romaguera, J. et al. Prepronociceptin-expressing neurons in the extended amygdala encode and promote rapid arousal responses to motivationally salient stimuli. Cell Rep. 33, 108362 (2020).

42. Rosbash, M. The implications of multiple circadian clock origins. PLoS Biol. 7, e62 (2009).

43. Rubio, F. J., Li, X., Liu, Q. R., Cimbro, R. \& Hope, B. T. Fluorescence activated cell sorting (FACS) and gene expression analysis of fos-expressing neurons from fresh and frozen rat brain tissue. J. Vis. Exp. 114, 54358 (2016).

44. Schmutz, I., Ripperger, J. A., Baeriswyl-Aebischer, S. \& Albrecht, U. The mammalian clock component PERIOD2 coordinates circadian output by interaction with nuclear receptors. Genes Dev. 24, 345-357 (2010).

45. Schwarz, J. M., Smith, S. H. \& Bilbo, S. D. FACS analysis of neuronal-glial interactions in the nucleus accumbens following morphine administration. Psychopharmacology 230, 525-535 (2013).

46. Song, A. J. \& Palmiter, R. D. Detecting and avoiding problems when using the cre-lox system. Trends Genet 34, 333-340 (2018).

47. Spanagel, R. et al. The clock gene Per2 influences the glutamatergic system and modulates alcohol consumption. Nat. Med. 11, 35-42 (2005).

48. Takahashi, J. S. Transcriptional architecture of the mammalian circadian clock. Nat. Rev. Genet. 18, 164-179 (2017).

49. Tso, C. F. et al. Astrocytes regulate daily rhythms in the suprachiasmatic nucleus and behavior. Curr. Biol. 27, 1055-1061 (2017).

50. Zhang, L. et al. A PERIOD3 variant causes a circadian phenotype and is associated with a seasonal mood trait. Proc. Natl. Acad. Sci. US A 113, E1536-E1544 (2016).

51. Zheng, B. et al. The mPer2 gene encodes a functional component of the mammalian circadian clock. Nature 400, 169-173 (1999).

52. Zhou, X. et al. Astrocyte, a promising target for mood disorder interventions. Front Mol. Neurosci. 12, 136 (2019).

53. Zhuo, L. et al. hGFAP-cre transgenic mice for manipulation of glial and neuronal function in vivo. Genesis 31, 85-94 (2001).

54. Zink, M., Vollmayr, B., Gebicke-Haerter, P. J. \& Henn, F. A. Reduced expression of GABA transporter GAT3 in helpless rats, an animal model of depression. Neurochem. Res. 34, 1584-1593 (2009).

\section{Acknowledgements}

We would like to acknowledge the invaluable advice from Prof. Jaclyn Schwarz from the University of Delaware on flow cytometry of brain tissue samples. S. Cattin and O. Coquoz from the Cell Analytics facility of the University of Fribourg are acknowledged for technical assistance and guidance for flow cytometry analysis and cell sorting experiments. We would also like to thank Dr. Jean-Charles Paterna from the Virus Vector Facility of the University of Zurich and ETH Zurich for providing valuable advice regarding the construction of viral vectors. We also thank A. Hayoz, S. Aebischer, and A. Sargsyan for technical support. AAV-pgk-Cre was a gift from Patrick Aebischer (Addgene viral prep \# 24593-AAVrg). This research was supported by the Swiss National Science Foundation (310030_184667/1).

\section{Author contributions}

Conceived and designed the experiments: T.M., J.S., A.K., U.A. Performed the experiments: T.M., J.A.R., J.S., M.S. Analyzed the data: T.M., J.A.R., J.S., M.S., U.A. Contributed reagents, materials, analysis tools: J.A.R., A.K., U.A. Wrote the paper: T.M., U.A.

\section{Competing interests}

The authors declare no competing interests.

\section{Additional information}

Supplementary Information The online version contains supplementary material available at https://doi.org/ 10.1038/s41598-021-91770-7.

Correspondence and requests for materials should be addressed to U.A.

Reprints and permissions information is available at www.nature.com/reprints.

Publisher's note Springer Nature remains neutral with regard to jurisdictional claims in published maps and institutional affiliations.

Open Access This article is licensed under a Creative Commons Attribution 4.0 International License, which permits use, sharing, adaptation, distribution and reproduction in any medium or format, as long as you give appropriate credit to the original author(s) and the source, provide a link to the Creative Commons licence, and indicate if changes were made. The images or other third party material in this article are included in the article's Creative Commons licence, unless indicated otherwise in a credit line to the material. If material is not included in the article's Creative Commons licence and your intended use is not permitted by statutory regulation or exceeds the permitted use, you will need to obtain permission directly from the copyright holder. To view a copy of this licence, visit http://creativecommons.org/licenses/by/4.0/.

(C) The Author(s) 2021 